\title{
Sensitivity pattern of Neisseria gonorrhoeae to penicillin and screening for B-lactamase production in Ibadan, Nigeria
}

\author{
A. O. OSObA, D. G. MOntefiore, A. O. SOgbetun, K. O. Alausa, \\ AND C. N. ANONG
}

From the Venereal Diseases and Treponematoses Research Laboratories, Department of Medical Microbiology, University College Hospital, Ibadan, Nigeria

SUMMARY The few reports from Africa on sensitivity determinations of Neisseria gonorrhoeae suggest that there is an increasing resistance in the strains, as has been found in other parts of the world. In the current study, the penicillin sensitivities and $\beta$-lactamase production of 80 consecutive strains of $N$. gonorrhoeae isolated from cases of acute urethritis in African men in Ibadan were studied. Of these strains, $17 \cdot 5 \%$ had a penicillin minimum inhibitory concentration (MIC) of $0.038 \mu \mathrm{g} / \mathrm{ml}$ or less and were considered as being 'fully sensitive'. However, $82.5 \%$ had an MIC of $0.075 \mu \mathrm{g}$ penicillin $/ \mathrm{ml}$ or more, and were considered as having 'diminished sensitivity' to penicillin. It was thought that this high incidence of relatively insensitive strains was owing to the sequential selection of these strains because of the easy availability and abuse of antimicrobial agents by the general population. Furthermore, 13 strains $(16 \cdot 25 \%)$ demonstrated high level resistance with MIC values of over $0.6 \mu \mathrm{g}$ penicillin per $\mathrm{ml}$ and it is probable that many of the strains will demonstrate multiresistance to the commonly misused antimicrobial agents. Consequently, treatment of patients harbouring these strains may present problems because of financial constraints of the health services in purchasing the appropriate antibiotics. Despite the high level resistance of the strains in an environment of intensive penicillin and ampicillin use, none of the strains studied showed any evidence of $\beta$-lactamase production. Nevertheless, continuous surveillance of $\beta$-lactamase production by the gonococcus is recommended in the larger medical centres in developing countries.

\section{Introduction}

The ever increasing prevalence of antibiotic resistant strains of Neisseria gonorrhoeae has been reported from all over the world (Willcox, 1972). Although penicillin still remains the drug of choice in the treatment of acute uncomplicated gonococcal infection, therapeutic failure with this drug is becoming more common. The recent reports of penicillinase-producing strains of $N$. gonorrhoeae questions the use of penicillin in the treatment of gonorrhoea (Ashford et al., 1976; Phillips, 1976).

Despite the lack of information, it is thought that Africa exemplifies the growing trend in penicillin

Address for reprints: A. O. Osoba, Venereal Diseases and Treponematoses Research Laboratories, Department of Medical Microbiology, University College Hospital, Ibadan, Nigeria

Received for publication 20 May 1977 insensitivity of the gonococcus. This has been confirmed by treatment failures and devastating chronic lesions of the gonococcus in Africa (Osoba and Alausa, 1976). Reports from Africa on the sensitivity pattern of the gonococcus to penicillin have been recorded from Uganda (Arya et al., 1973), Ethiopia (Plorde et al., 1973), Kenya (Verhagen et al., 1971), and South Africa (Finlayson et al., 1974). These reports showed a decreased sensitivity to penicillin ranging from 20 to $80 \%$ of the various strains tested.

More recently, one of the earliest strains of $\beta$-lactamase-producing gonococci was thought to have originated from West Africa (Phillips, 1976; Arya, personal communication). We therefore thought it worthwhile to study the penicillin sensitivity and $\beta$-lactamase production of strains of $N$. gonorrhoeae isolated at the University College Hospital, Ibadan, Nigeria. 


\section{Materials and methods}

Strains of $N$. gonorrhoeae were obtained from 80 consecutive cases of acute purulent gonococcal urethritis in African men presenting at the Special Treatment Clinic, University College Hospital, Ibadan, Nigeria. The specimens were obtained by passing sterile cotton-wool swabs into the anterior urethra and plating directly on to Thayer-Martin medium (Oxoid) and chocolate agar. The plates were then incubated in candle extinction jars at $37 \mathrm{C}$ for between 24 and 48 hours. Typical colonies were picked and Gram stained. All Gram-negative cocci were then further identified after subcultures on brain heart infusion agar (BHIA) (Oxoid) by the oxidase reaction and fermentation reaction in serumfree agar sugar medium (Flynn and Waitkins, 1972). Strains were identified as being $N$. gonorrhoeae only if they were oxidase-positive and fermented glucose but not sucrose or maltose.

MINIMUM INHIBITORY CONCENTRATION

(MIC) DETERMINATION

Serial twofold dilutions of penicillin $\mathrm{G}$ (Crystapen) were added to BHIA cooled to $50 \mathrm{C}$ to give final concentrations of $1 \cdot 2,0 \cdot 6,0 \cdot 3,0 \cdot 15,0 \cdot 075,0 \cdot 038$, $0.019,0.01$, and $0.005 \mu \mathrm{g}$ penicillin $/ \mathrm{ml}$. The agar was allowed to set and the plates were then dried in an incubator at $40^{\circ} \mathrm{C}$ for 30 minutes and used on the day of preparation.

Several colonies of the strain to be tested were picked from a 48-hour culture on BHIA and emulsified in saline. The concentration was adjusted visually to a No.05 McFarland standard. This suspension contained between $10^{7}$ and $10^{8}$ organisms $/ \mathrm{ml}$.

An even suspension of the organisms was obtained by agitation on a vortex mixer for about 10 seconds. Using a standard platinum loop, $0.001 \mathrm{ml}$ of the suspension was inoculated on to the antibioticcontaining plates. These were then incubated in an extinction jar at $37 \mathrm{C}$ for 18 hours and the tests read as MICs. The lowest concentration of penicillin which completely inhibited the growth of the test organism or caused a decrease of more than $95 \%$ in the growth of the organisms was regarded as the MIC for that strain.

A control plate without an antibiotic and a reference strain of Staphylococcus aureus with an MIC of $0.036 \mu \mathrm{g}$ penicillin $/ \mathrm{ml}$ were included in each test. Reference strains of the World Health Organisation of $N$. gonorrhoea III, V, and VII were obtained from Dr Alice Reyn at the Statens Serum Institut, Copenhagen. Using the above method the MIC from the reference strains did not differ significantly from the figures obtained at the WHO reference laboratory.
CHROMOGENIC CEPHALOSPORIN TEST

$5 \mathrm{mg}$ of chromogenic cephalosporin compound $87 / 312$ (Glaxo) were dissolved in $0.5 \mathrm{ml}$ of dimethyl sulphoxide and the solution was made up to $10 \mathrm{ml}$ with $0.1 \mathrm{~mol} / 1$ phosphate buffer $\mathrm{pH} 7 \cdot 0$. The solution was used either freshly prepared or after storage at $4 \mathrm{C}$ for not longer than one week. To test for $\beta$-lactamase production, the solution was dropped into confluent growth of the $N$. gonorrhoeae strain under test and observed for the development of a red colour both immediately and after a further 30 minutes' incubation at $36^{\circ} \mathrm{C}$. A control culture of penicillinase-producing $S$. aureus was used to ensure that the reagent was working satisfactorily each time it was used.

\section{Results}

The sensitivities to penicillin of the 80 strains of $N$. gonorrhoeae are illustrated in the Figure. Fourteen strains $(17.5 \%)$ had MIC values to penicillin of $0.038 \mu \mathrm{g} / \mathrm{ml}$ or less. These were considered as being 'fully sensitive'. Fifty-three strains $(66.25 \%)$ had MIC values of 0.075 to $0.3 \mu \mathrm{g} / \mathrm{ml}$ penicillin. These we considered to have a 'reduced sensitivity' to penicillin. Thirteen strains $(16 \cdot 25 \%)$ had MIC values of over $0.6 \mu \mathrm{g}$ penicillin $/ \mathrm{ml}$. These were considered as being 'highly resistant'.

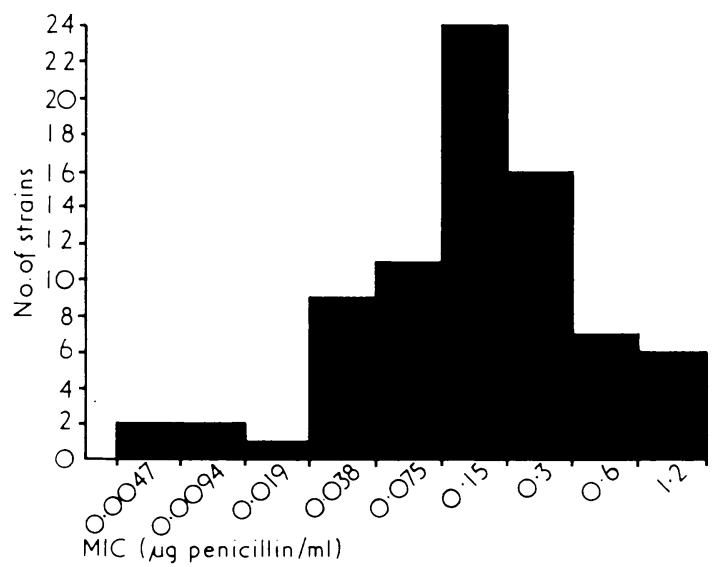

Figure The sensitivities to penicillin of the 80 strains of N. gonorrhoeae.

All the highly resistant strains were tested and gave a negative result on the chromogenic cephalosporin test indicating that none of them produced penicillinase. 


\section{Discussion}

Reports about antimicrobial drug resistance by strains of $N$. gonorrhoeae started to appear in 1944 (Reyn et al., 1958). Since then the impression that there was a progressive increase of gonococcal resistance to antibiotics, especially to penicillin, has been confirmed (Amies, 1969; Willcox, 1970, 1972).

Although the situation in the African region is not as well defined as in other countries, the little information available indicates that the world-wide pattern also applies to this continent. In West Africa, although $99 \%$ of 74 gonococcal strains were found to be 'fully sensitive' in 1961 (World Health Organisation, 1963), Clarke (1964) working in Lagos, encountered treatment failure in $21.4 \%$ of patients who received procaine penicillin 2.4 megaunits daily for three consecutive days although no sensitivity tests were performed. In Yaunde, Cameroons, Millan and Huet (1970), using a disc diffusion method, reported that 10 of their 18 gonococcal strains were 'sensitive' in penicillin. Osoba (1972), using the disc diffusion technique, found 19 of 185 strains of gonococci isolated in Ibadan were resistant to 1.5 units of penicillin. Three of these strains came from prostitutes taking prophylactic antibiotics.

In Ethiopia, Plorde et al. (1973) found 234 (50\%) strains from Addis Ababa had an MIC value to penicillin of $0.036 \mu \mathrm{g} / \mathrm{ml}$ or less. Increasing resistance of the gonococcus to penicillin has been demonstrated in East Africa by Phillips et al. (1969) who found that $193(70 \%)$ strains were resistant to $0.06 \mu \mathrm{g} / \mathrm{ml}$ penicillin or less, and Arya and Phillips (1970) who reported that $173(80 \%)$ strains showed diminished sensitivity to penicillin.

We found $82.5 \%$ of our strains showed diminished sensitivity to penicillin. This is comparable with the East African figures and with $85 \%$ reported in the Far East (Keys et al., 1969), but is higher than the British figures of $17 \cdot 6 \%$ (Shannon et al., 1975) and 30\% (Shahidulla and Greaves, 1975). It is also higher than the figures reported from Bombay (Moses et al., 1971) and the 70\% reported from the USA (Jaffe et al., 1976). The level of gonococcal penicillin resistance in our series therefore ranks as one of the highest in the world. When the figures obtained in the current study are compared with those reported in 1961 (World Health Organisation, 1963) it appears that penicillininsensitive strains are being rapidly and sequentially selected, particularly during the last 15 years.

Several reasons can be found for this high incidence. The main one is the easy availability and abuse of antimicrobial agents by the general population. It has been shown that by improving the results of treatment of gonorrhoea by increasing the dosage of penicillin lowers the prevalence of partially resistant strains (Morton 1963; Olsen and Lomholt, 1969). However, because of the inadequate medical facilities and a general misconception of antimicrobial agents by patients, there is reckless selfmedication. Not only are antibiotics taken prophylactically after sexual intercourse with casual consorts, but even if symptoms of acute urethritis develop, subcurative and ineffective doses of these agents are taken haphazardly for varying periods before a proper medical opinion is sought.

With a gonorrhoea infection rate of $15.8 \%$ among prostitutes and $17 \%$ among female hospital patients in Ibadan (Osoba, 1972), and widespread promiscuity, this high rate of penicillin resistance in strains of the gonococcus poses a great economic problem as more expensive antimicrobial agents will have to be used to treat these patients. More alarming still is the evidence obtained in Kenya by Verhagen et al. (1971) that the reservoir of relatively resistant strains in a tropical country is in the average rural population. Nigeria, has a population of about 70 million of whom about $75 \%$ live in rural areas, so the reservoir of resistant strains may be one of considerable proportions, if the hypothesis is sustained.

In the current study, 53 strains $(66.25 \%)$ had a reduced sensitivity to penicillin but 13 strains $(16 \cdot 25 \%)$ had high level resistance with MIC values over $0.6 \mu \mathrm{g}$ penicillin per $\mathrm{ml}$ (Figure). This alarming finding supports the suggestion of Verhagen et al. (1971) that more high level relatively resistant strains occur in developing countries than elsewhere.

We did not test the sensitivity of the strains isolated in this study against the commonly misused antibiotics - such as, sulphonamides, streptomycin, tetracycline, ampicillin, and chloramphenicol-but judging by experiences elsewhere (Verhagen et al. 1971), it is probable that many of the strains isolated here would demonstrate multiresistance to antimicrobials readily available without prescription here, as in many developing countries. It is hoped that governments will be able to control the availability of these drugs to the public, if only to check the rapid sequential selection of resistant strains.

None of our strains produced $\beta$-lactamase. This is surprising as the $\beta$-lactamase gene is plasmid-borne. If it is assumed that this plasmid is transferred from other bacteria in an area of intensive penicillin and ampicillin use, one would expect that the indiscriminate use of antibiotics would give an environment favouring such genetic change. However, the number of our isolates from a single clinic may not be representative of the gonococcal population in 
the whole country. The continuous surveillance of $\beta$-lactamase production by the gonococcus is obviously a priority in Nigeria as in many developing countries, since the cost and availability of other antibiotics may create considerable problems in treating cases, with resultant failure of control measures; these at present, are inadequate in many developing countries.

There is therefore a need to screen rural communities in tropical Africa to determine the extent of the reservoir of gonococcal infection and to ascertain the sensitivity pattern of the local strains to the commonly available antimicrobial agents. Financial constraints and inadequate facilities are common in these areas, and these together with promiscuity, polygamy, and ignorance constitute the major reasons for failure to control the rising incidence of gonococcal infection in many developing countries.

We wish to record our gratitude to Dr R. B. Sykes, Glaxo Research Ltd, for supplying Chromogenic Cephalosporin Compound $87 / 312$ and to $\mathrm{Mr} \mathrm{J}$. Ochei of the Department of Medical Microbiology, University College Hospital, Ibadan, for his technical assistance. This study was supported by a WHO grant which is gratefully acknowledged.

\section{References}

Amies, C. R. (1969). Sensitivity of Neisseria gonorrhoeae to penicillin and other antibiotics. British Journal of Venereal Diseases, 45, 216-222.

Arya, O. P., Nsanzumuhire, H., and Taber, S. R. (1973). Clinical, cultural and demographic aspects of gonorrhoea in a rural community in Uganda. Bulletin of the World Health Organisation, 49, 587-595.

Arya, O. P., and Phillips, I. (1970). Antibiotic sensitivity of gonococci and treatment of gonorrhoea in Uganda. British Journal of Venereal Diseases, 46, 149-152.

Ashford, W. A., Golash, R. G., and Hemming, V. G. (1976). Penicillinase-producing Neisseria gonorrhoeae. Lancet, 2, 657-658.

Clarke, G. H. V. (1964). Comparison of different antibiotics in the treatment of acute gonorrhoea in African negroes. British Journal of Venereal Diseases, 40, 122-124.

Finlayson, M. H., Gibbs, B., and Brede, H. D. (1974). Diagnosis and incidence of Neisseria gonorrhoeae in Cape coloured females in Western Cape: Laboratory aspects. South African Medical Journal, 48, 259-260.
Flynn, J., and Waitkins, S. A. (1972). A serum-free medium for testing fermentation reactions in Neisseria gonorrhoeae. Journal of Clinical Pathology, 25, 525-527.

Jaffe, H. W., Biddle, J. W., Thornsberry, C., Johnson, R. E., Kaufman, R. E., Reynolds, G. H., Wiesner, P. J., and the Co-operative Study Group. (1976). Gonorrhea therapy monitoring: In vitro antibiotic susceptibility and its correlation with treatment results. New England Journal of Medicine, 294, 5-9.

Keys, T. F., Halverson, C. W., and Clarke, E. J. (1969). Single dose treatment of gonorrhea with selected antibiotic agents. Journal of the American Medical Association, 210, 857-861.

Millan, J., and Huet, M. (1970). A propos de l'étude de la sensibilité aux antibiotiques de souches de gonocoques isolées à Yaoundé: Considérations épidémiologiques bactériologiques et thérapeutiques. Bulletin de la Société de pathologie exotique et de ses filiales, 63, 642-652.

Morton, R. S. (1963). Epidemiology of gonorrhoea: A departure from the typical incidence picture. British Journal of Venereal Diseases, 39, 105-108.

Moses, J. M., Desai, M. S., Bhosle, C. B., and Trasi, M. S. (1971) Present pattern of sensitivity of gonococcal strains isolated in Bombay. British Journal of Venereal Diseases, 47, 273-278.

Olsen, G. A., and Lomholt, G. (1969). Gonorrhoea treated by a combination of probenecid and sodium penicillin G. British Journal of Venereal Diseases, 45, 144-148.

Osoba, A. O. (1972). Epidemiology of urethritis in Ibadan. British Journal of Venereal Diseases, 48, 116-120.

Osoba, A. O., and Alausa, K. O. (1976). Gonococcal urethral stricture and watering-can perineum. British Journal of Venereal Diseases, 52, 387-393.

Phillips, I. (1976). Beta-lactamase-producing penicillin-resistant gonococcus. Lancet, 2, 656-657.

Phillips, I., Fernandes, R., Pirani, A. A., and Wagaine, D. (1969). Antibiotic sensitivity of gonococci in Kampala. East African Medical Journal, 46, 38-45.

Plorde, J. J., Kidan, T. G., and Wright, L. J. (1973). Penicillin sensitivity of gonococci in Ethiopia. British Journal of Venereal Diseases, 49, 260-262.

Reyn, A., Korner, B., and Bentzon, M. W. (1958). Effects of penicillin, streptomycin and tetracycline on $N$. gonorrhoeae isolated in 1944 and in 1957. British Journal of Venereal Diseases, 34, 227-239.

Shahidullah, M., and Greaves, P. W. (1975). Minimum inhibitory concentrations of penicillin and minocycline for 300 isolates of N. gonorrhoeae. British Journal of Venereal Diseases, 51, 265-266.

Shannon, R., Hedges, A. J., and Edwards, R. J. (1975). Distribution of levels of penicillin resistance among freshly isolated strains of $N$. gonorrhoeae. Application of a novel sensitivity assay. British Journal of Venereal Diseases, 51, 246-250.

Verhagen, A. R., Vander Ham, M., Heimans, A. L., Kranendonk, O., and Maina, A. M. (1971). Diminished antibiotic sensitivity of Neisseria gonorrhoeae in urban and rural areas in Kenya. Bulletin of the World Health Organisation, 45, 707-717.

Willcox, R. R. (1970). A survey of problems in the antibiotic treatment of gonorrhoea. With special reference to South East Asia. British Journal of Venereal Diseases, 46, 217-242.

Willcox, R. R. (1972). A world-wide view of venereal diseases. British Journal of Venereal Diseases, 48, 163-176.

World Health Organisation (1963). Expert Committee on Gonococcal Infections. Technical Report Series, No. 262, WHO: Geneva. 